\title{
Prevalence and diagnostic distribution of medically unexplained painful somatic symptoms across 57I major depressed outpatients
}

This article was published in the following Dove Press journal:

Neuropsychiatric Disease and Treatment

19 April 201 I

Number of times this article has been viewed

\author{
Michele Fornaro' \\ Icro Maremmani ${ }^{2,3}$ \\ Pier Luigi Canonico ${ }^{4}$ \\ Paolo Carbonatto ${ }^{5}$ \\ Claudio Mencacci ${ }^{6}$ \\ Giovanni Muscettola ${ }^{7}$ \\ Luca Pani ${ }^{8}$ \\ Riccardo Torta 9 \\ Claudio Vampini ${ }^{10}$ \\ Fabio Parazzini"l \\ Arina Dumitriu' ${ }^{12}$ \\ Giulio Perugi ${ }^{2,3}$ \\ 'Department of Neurosciences, \\ Section of Psychiatry, University of \\ Genova, Genoa, Italy; ${ }^{2}$ Dipartimento \\ di Psichiatria Università di Pisa, Pisa, \\ Italy; ${ }^{3}$ Institute of Behavioural Sciences \\ “G. De Lisio", Pisa, Italy; ${ }^{4}$ Università di \\ Novara, Novara, Italy; ${ }^{5}$ Societa' Italiana \\ di Medicina Generale (SIMG) Turin, \\ Italy; ${ }^{6}$ Ospedale Fatebenefratelli, Milan, \\ Italy; ${ }^{7}$ Università di Napoli, Naples, \\ Italy; ${ }^{8}$ Istituto di Neurogenetica e \\ Neurofarmacologia, CNR, Cagliari, \\ Italy; ${ }^{9}$ Ospedale Molinette, Turin, Italy; \\ ${ }^{10}$ Ospedale Maggiore, Verona, Italy; \\ "GPA net, Milan, Italy; ${ }^{12}$ Boehringer \\ Ingelheim S.p.A., Milan, Italy
}

Correspondence: Michele Fornaro Largo R. Benzi n. 16, Ospedale San Martino, Clinica Psichiatrica Universitaria, ZIP 16100, Genova, Italy

Tel $+39010353768 \mid$

Fax +393537669

Email dott.fornaro@gmail.com
Objective: To assess the prevalence and distribution of medically unexplained painful somatic symptoms (PSSs) versus nonpainful somatic symptoms (NPSSs) in patients diagnosed with major depressive episode (MDE).

Method: A total of 571 outpatients diagnosed with MDE according to DSM-IV-TR criteria were consecutively enrolled into a cross-sectional, multicentric, observational study over a period of 7 months. Subjects were evaluated by means of the ad hoc validated 30-item Somatic Symptoms Checklist (SSCL-30) and Zung's questionnaires for depression and anxiety. The 32-item Hypomania Checklist (HCL-32) was also administered in order to explore any eventual association of PSSs or NPSSs with sub-threshold (DSM-IV-TR [Diagnostic and Statistical Manual of Mental Disorders, Fourth Edition, Text Revision] not recognized) bipolar disorder (BD).

Results: In our sample, just 183 patients (32\%) did not report painful somatic symptoms (NPSSs). Of these, 90 patients $(15.76 \%$ ) had no somatic symptoms at all. The remaining 388 (68\%) had at least one PSS being subdivided as follows: 248 (43\%) had one or two PSSs, while $140(25 \%)$ experienced two or more. Patients with at least one PSS also reported a greater number of nonpainful somatic symptoms than NPSS. Bipolar patients (associated with higher HCL-32 scores) were less represented across PSS cases than NPSS subjects. Conversely, females were more prone to having a higher number of total somatic symptoms (and bipolar features).

Conclusion: PSSs are common in patients with MDE, especially among those patients reporting fewer somatic symptoms in general as opposed to those patients who exhibit more somatic symptoms (both PSSs and NPSSs) with lower relative number of PSSs. A major therapeutic implication is that antidepressant monotherapy could be used with more confidence in unexplained PSS patients than in NPSS patients because of the latter group's lower frequency of (sub)-threshold bipolar features.

Keywords: major depressive episode, MDE, bipolar disorder, BD

\section{Introduction}

Major depressive episode (MDE) represents a multidimensional condition possibly exhibiting with a wide range of clinical pictures. Frequently, clinical presentations may include the presence of painful (PSSs) as well as nonpainful somatic symptoms (NPSSs). ${ }^{1}$ Nonetheless, somatic symptoms in the course of MDE often go underestimated $^{2}$ despite their impact on the course of illness and treatment choices. In fact, when inappropriately recognized and treated, (medically unexplained) somatic symptoms - considered as symptoms otherwise not referable to nonpsychiatric disturbances - are associated with poor prognosis, higher risk for residual 
symptomatology, as well as increased risk for relapse and recurrence. ${ }^{3-6}$ Hence, the need for their appreciation and proper recognition is unquestionable. Furthermore, MDE could frequently represent the main or even the only manifestation of bipolar disorder (BD) lasting for many years prior to the onset of frank (hypo)-mania. Thus, the importance of early appropriate recognition and management of depressive features, eventually pointing out a bipolar diathesis, should be considered mandatory in order to reduce the chance of iatrogenic effects as the antidepressant-induced resistance to treatment or cyclicity of the disorder. ${ }^{7}$

The aim of the present study is to assess the distribution of PSSs and NPSSs on MDE (including both single and recurrent episodes) and to explore their possible association with bipolarity assessed according to Diagnostic and Statistical Manual of Mental Disorders, Fourth Edition, Text Revision [DSM-IV-TR] criteria (full-threshold BD) and with the 32-item Hypomania Checklist (HCL-32) rating scale ${ }^{8}$ (for sub-threshold bipolarity).

\section{Methods}

The present study is based on a post-hoc partial analysis of the database collected for a previous study, including 571 consecutive patients with a diagnosis of MDE, according to DSM-IV-TR, that were enrolled in a large cross-sectional, multicentre, observational study (COME TO ME). ${ }^{9}$ The study involved 30 psychiatric outpatient facilities, distributed throughout Italy, investigating the prevalence of medically unexplained somatic symptoms in the course of MDE, which resulted in severe depressive symptomatology, high rates of misdiagnosis, and improper treatment and health resource utilization.

According to the observational nature of the protocol, routine medical procedures were not modified. The Ethics Committee of each center approved the study protocol in compliance with the Italian Ministerial Bulletin issued on September 2, 2002 regarding observational studies. All patients gave their informed consent concerning handling and use of the data collected during the course of the study. Finally, the study was sponsored by Boehringer Ingelheim, Italy.

\section{Study population}

Subjects referred to the selected centers between December 2006 and July 2007 were screened for inclusion in the study. Patients were recruited consecutively, according to the following inclusion criteria: 1) both genders, aged 18-75 years; 2) diagnosis of major depressive episode according to DSM-IV-TR (major depressive disorder, recurrent major depression, depressive episode in course of type I and II bipolar disorder, depression not otherwise specified (NOS); and 3 ) ability to complete the self- and hetero-administered questionnaires. The exclusion criteria were: 1) comorbidity with schizophrenia and other psychotic disturbances, and 2) current relevant physical illnesses.

The study included 571 depressed outpatients; 8 (1.4\%) of them had incomplete information on demographic and clinical variables. The study population therefore consisted of 563 eligible subjects, with a mean number of patients of 18.7 (range 5-56) for each center; 376 subjects (66.8\%) were females and 187 (33.2\%) males; 251 subjects (44.5\%) were aged $18-50$ years, and $313(55.5 \%)$ aged over 50 , with mean age of 51 (standard deviation $=13$ ). The level of education was higher than 11 years for $245(43.5 \%)$ patients, and most of them $(n=331,58.8 \%)$ were married.

\section{Diagnostic procedure and symptom assessment}

Individuals were assessed by psychiatrists with extensive clinical experience in the diagnosis and treatment of mood disorders. Diagnosis of MDE (major depressive disorder, recurrent major depression, depressive episode type I and II bipolar disorder; depression NOS) was made according to DSM-IV-TR criteria.

An anonymous datasheet was used to collect the following information: demographic status, lifestyle, surgical, pathological, and pharmacological case history, presence and characterization of somatic symptoms and related delay in major depression diagnosis and treatment, and consumption of health resources within the 6 months preceding enrollment. All of the information was gathered directly by the patient with the help of significant others and medical records.

HCL- $32^{8}$ was administered for the evaluation of lifetime manic or hypomanic features, and severity of depressive and anxious symptomatology was self-evaluated by means of the Zung's questionnaires for depression and anxiety. ${ }^{10,11}$

\section{Statistical analysis}

Comparative analysis for familial, epidemiological, clinical, and course characteristics of subgroups were conducted using the Student's $t$-test for the dimensional variables (Mann-Whitney $U$-test, when appropriate) and the chi-square analysis for those categories (Fisher exact-test, when appropriate). We used a Bonferroni's significance of $P=0.001$ level corrected for multiple comparisons. Given the exploratory nature of the study, we set significance at level of 0.5 , two 
tailed, in order to detect potentially clinically meaningful associations.

\section{Results}

Among the 571 MDE outpatients of our sample, only 183 patients (32\%) did not report unexplained PSS (NPSSs), including 90 subjects $(15.76 \%)$ who reported no somatic symptoms at all. The other $388(68 \%)$ patients had at least one PSS. Among this latter group, 248 (43\%) had one or two, while 140 (25\%) experienced two or more. The patients with at least one PSS who also reported associated NPSSs also showed a higher total number of NPSSs compared with the patients without PSSs, with a mean NPSSs number of $4.3 \pm 3.3$ versus $2.3 \pm 1.7(t=9.2, P=0.0001)$.

Headache was the most frequently reported PSS $(n=220$, $38.5 \%$ ), followed by osteo-articular pain ( $\mathrm{n}=200,35 \%)$, and gastralgia ( $\mathrm{n}=102,17.9 \%)$; concerning NPSSs, asthenia was the far more represented symptom $(n=368,64.4 \%)$, followed by insomnia $(n=237,41.5 \%)$, tenseness $(n=227,39.8)$, palpitations $(n=190,33.3 \%)$, dizziness $(n=114,20 \%)$, decreased libido $(\mathrm{n}=108,19 \%)$, tremor $(\mathrm{n}=108,19 \%)$, decreased appetite $(\mathrm{n}=105,18.4 \%)$, constipation $(\mathrm{n}=89,15.6 \%)$, dyspnea $(\mathrm{n}=89,15.6 \%)$, and sweating $(\mathrm{n}=86,15.1)($ Table 1$)$.

Comparing the three groups (NPSS, one or two PSSs, and three or more PSSs), no differences were found concerning demographic features except for gender distribution. Females were more represented among patients with two or more PSSs, compared with groups with one PSS and NPSS (Table 2).

Concerning diagnostic distribution, bipolar depression was more frequent among patients with NPSS than in patients with PSSs $(P=0.001)$; also, the higher the number of PSSs, the lower the chance of bipolar diagnosis.

In contrast, depression NOS showed an inverse trend. As expected, Zung's depression and anxiety scores greater than 50 were more represented among the group of patients with three or more PSSs than in the other two groups. Remarkably, patients with three or more PSSs were less frequently represented among those having an HCL-32 total score greater than 14 (the cutoff diagnostic value), while a HCL-32 total score lower than 14 was more associated with NPSSs (Table 2).

\section{Discussion}

In interpreting our results, some methodological limitations must first be taken into account. Although recorded, pharmacological treatments were not considered as potentially influencing the perception of somatic pain. Yet, it must be noted
Table I Medically unexplained somatic symptoms in 57I major depressed patients: frequency distribution

\begin{tabular}{|c|c|c|}
\hline Symptom $^{a}$ & $\mathbf{N}$ & $\%$ \\
\hline \multicolumn{3}{|l|}{ PSSs } \\
\hline 3. Headache & 220 & 38.5 \\
\hline 13. Osteoarticular pain & 200 & 35.0 \\
\hline 17. Gastralgia & 102 & 17.9 \\
\hline 9. Dyspepsia & 83 & 14.5 \\
\hline 12. Abdominal pain & 75 & 13.1 \\
\hline I5. Transient pain & 68 & 11.9 \\
\hline 7. Dysphagia & 41 & 7.2 \\
\hline 14. Chest pain & 41 & 7.2 \\
\hline 2I. Itching & 36 & 6.3 \\
\hline II. Dysuria & 22 & 3.9 \\
\hline 8. Dysmenorrhea & 18 & 3.2 \\
\hline \multicolumn{3}{|l|}{ NPSSs } \\
\hline 2. Asthenia & 368 & 64.4 \\
\hline 19. Insomnia & 237 & 41.5 \\
\hline 25. Tenseness & 227 & 39.8 \\
\hline 20. Palpitations & 190 & 33.3 \\
\hline 30. Dizziness & 114 & 20.0 \\
\hline 6. Decreased libido & 108 & 18.9 \\
\hline 27. Tremor & 108 & 18.9 \\
\hline 18. Decreased appetite & 105 & 18.4 \\
\hline 4. Constipation & 89 & 15.6 \\
\hline 10. Dyspnea & 89 & 15.6 \\
\hline 24. Sweating & 86 & 15.1 \\
\hline 23. Drowsiness & 78 & 13.7 \\
\hline 29. Flushing & 77 & 13.5 \\
\hline I. Aerophagia & 61 & 10.7 \\
\hline 28. Tenesmus & 53 & 9.3 \\
\hline 5. Diarrhea & 37 & 6.5 \\
\hline I6. Flatulence & 35 & 6.1 \\
\hline 26. Cough & 26 & 4.6 \\
\hline 22. Hiccups & 10 & 1.9 \\
\hline
\end{tabular}

Note: ${ }^{2}$ The numbers refer to the items of the SSCL-30 scale.

Abbreviations: NPSS, painless somatic symptoms; PSS, painful somatic symptoms; SSCL-30, 30-item Somatic Symptoms Checklist.

that they might have influenced the pharmacological choices of the therapist. As expected, somatic symptoms were a frequent finding in the course of depression, especially for female patients. Nonetheless, avoidance of a systematic recording of the clinical history of the subjects might have further limited the validity of our results. This seems particularly true concerning the lack of information about the number and severity of previous episodes, if any, and the eventual presence of previous hospitalizations and/or suicidal attempts. Also, the anxiety/ depression scores appear driven by endorsing the somatic items in the SSCL-30 scale, thus limiting the reliability of this finding, especially considering the absence of accurate records of anxious comorbidities (ie, somatoform disorder).

The fact that medically unexplained somatic complaints were essentially not associated with bipolarity is also a remarkable result since it was initially expected to be related 
Table 2 Comparison between depressed patients with painless and painful somatic symptoms

\begin{tabular}{|c|c|c|c|c|c|}
\hline & $\begin{array}{l}\text { NPSSs } \\
N=183\end{array}$ & $\begin{array}{l}\text { PSSs I-2 } \\
N=248\end{array}$ & $\begin{array}{l}\text { PSSs }>2 \\
N=\mid 40\end{array}$ & Chi (df) & $P$ \\
\hline & $\mathbf{N}(\%)$ & $\mathbf{N}(\%)$ & $\mathbf{N}(\%)$ & & \\
\hline \multicolumn{6}{|l|}{ Age, years } \\
\hline$\leq 45$ & $66(36.1)$ & $82(33.1)$ & $39(27.9)$ & & \\
\hline $46-58$ & $66(36.1)$ & $73(29.4)$ & $53(37.9)$ & $6.93(4)$ & 0.139 \\
\hline$>58$ & 51 (27.9) & 93 (37.5) & $48(34.3)$ & & \\
\hline \multicolumn{6}{|l|}{ Gender } \\
\hline Female & $107(58.5)$ & $168(67.7)$ & $108(77.1)$ & $|2.6|(2)$ & 0.001 \\
\hline Male & $76(4 \mid .5)$ & $80(32.3)$ & $32(22.9)$ & & \\
\hline \multicolumn{6}{|l|}{ Diagnostic distribution } \\
\hline Major depression & $64(35.0)$ & $77(31.0)$ & $56(40.0)$ & & \\
\hline Major recurrent depression & $66(36.1)$ & 9I (36.7) & $58(4 \mid .4)$ & $26.91(6)$ & 0.00015 \\
\hline Bipolar depression & $50(27.3)$ & $56(22.6)$ & $13(9.3)$ & & \\
\hline Depression NOS & $3(1.6)$ & $24(9.7)$ & $13(9.3)$ & & \\
\hline \multicolumn{6}{|l|}{ Zung depression } \\
\hline$<50$ & $85(46.4)$ & $85(34.3)$ & $28(20.0)$ & $24.52(2)$ & 0.00000 \\
\hline$\geq 50$ & $98(53.6)$ & $163(65.7)$ & $112(80.0)$ & & \\
\hline \multicolumn{6}{|l|}{ Zung anxiety } \\
\hline$<50$ & $138(75.4)$ & $149(60.1)$ & $53(37.9)$ & $46.48(2)$ & 0.00000 \\
\hline$\geq 50$ & $45(24.6)$ & $99(39.9)$ & $87(62.1)$ & & \\
\hline \multicolumn{6}{|l|}{ Hypomania checklist } \\
\hline $0-14$ & $101(62.2)$ & $|4|(67.1)$ & 95 (77.2) & $8.31(2)$ & 0.015 \\
\hline$>14$ & $64(38.8)$ & $69(32.9)$ & $28(22.8)$ & & \\
\hline
\end{tabular}

Abbreviations: NOS, not otherwise specified; NPSS, painless somatic symptoms; PSS, painful somatic symptoms.

to the "excitement" of mania. Rather, the total number of somatic symptoms (including both PSSs and NPSSs) was higher among those with a bipolar diagnosis. These findings indicate that PSSs could be less represented in "bipolar" (HCL-32 total score $\geq 14$ ) MDEs rather than unipolar MDEs. A major therapeutic implication is that antidepressant monotherapy could be used with more confidence in unexplained PSS patients than in NPSS patients because of the latter group's lower frequency of (sub)-threshold bipolar features. Specifically, the use of SNRIs (serotonin norepinephrine reuptake inhibitors) or the choice of tricyclic antidepressants (TCAs), especially the more pro-norepinephrinergic secondary amines, may be preferred as hypothetically more somatic-symptoms-oriented therapies for those with PSSs (found to have also a higher total number of somatic symptoms in general). Furthermore, TCAs and SNRIs might be less prone to induce (hypo) manic switches in PSS patients compared with (bipolar) NPSSs.

Finally, stating the strong limits of our exploration, further longitudinal, more accurate investigations are needed in order to confirm this merely preliminary report.

\section{Acknowledgments}

The authors sincerely acknowledge the COME TO ME Study Group: Agrimi Emilia, MD, U.O. Psichiatria 29,
"Istituti Ospitalieri", Cremona, Italy; Amati Amato, MD, Policlinico "Mater Domini", Clinica Psichiatrica Università di Catanzaro, Italy; Bogetto Filippo, MD, Azienda Sanitaria Ospedaliera Molinette, Dipartimento di Neuroscienze Università di Torino, Italy; Bellomo Antonello, MD, U.O. Psichiatria Ospedale Riuniti di Foggia, Italy; Carpiniello Bernardo, MD, Clinica Psichiatrica Università degli Studi di Cagliari, Italy; Castrogiovanni Paolo, MD, Policlinico Universitario "Le Scotte", Clinica Psichiatrica Università di Siena, Italy; Colotto Antonio, MD, U.O. Psichiatria USL della Valle di Aosta, Aosta, Italy; Costa Raffaele, MD, U.O. Salute Mentale DSM ASL “Genovese”, Genova, Italy; Di Fiorino Mario, MD, Reparto di Psichiatria, Ospedale della Versilia, Viareggio (Lucca), Italy; Federico Tommaso, MD, ASL 3 - Dipartimento di Salute Mentale, Catania, Italy; Meduri Mario, MD, Clinica Psichiatrica Università degli Studi di Messina, Italy; Minnai Gian Paolo, MD, Ospedale S Martino, Dipartimento di Salute Mentale, Oristano, Italy; Nardini Marcello, MD, Dipartimento Scienze Neurologiche Psichiatriche Universitarie, Bari, Italy; Aguglia Eugenio, MD, U.O. di Clinica Psichiatrica Università degli Studi di Catania, Italy; Placidi Gian Franco, MD, Ospedale Careggi, Dipartimento di Neuropsichiatria Università degli Studi di Firenze, Italy; Nivoli Giancarlo, MD, Clinica Psichiatrica Università degli Studi, Sassari, Italy; Pellegrino Ferdinando, MD, U.O. 
DSM ASL "Salerno 1" - Distretto 5C, Salerno, Italy; Sartore Luigi, MD, ASL 20 - Struttura Complessa Salute Mentale, Alessandria, Italy; Sciarretta Antonio, MD, DSM, ASL RMG - Ambulatorio Psichiatrico, Tivoli, Italy; Varia Salvatore, MD, DSM modulo $\mathrm{N}^{\circ} 7$, ASL Palermo, Italy; Viola Marcello, MD, Centro Coordinamento Salute Mentale, Brindisi, Italy.

\section{Disclosure}

The database-source COME TO ME study was supported by Boehringer Ingelheim S.p.A. Italy.

\section{References}

1. American Psychiatric Association, ed. Diagnostic and Statistical Manual for Mental Disorders, Fourth Edition, Text Revision (DSM-IV-TR). Washington, D.C.: American Psychiatric Association; 2000.

2. Henriques SG, Fraguas R, Iosifescu DV, et al. Recognition of depressive symptoms by physicians. Clinics (Sao Paulo). 2009;64(7): 629-635.
3. Karp JF, Scott J, Houck P, Reynolds CF 3rd, Kupfer DJ, Frank E. Pain predicts longer time to remission during treatment of recurrent depression. J Clin Psychiatry. 2005;66(5):591-597.

4. Greden JF. Physical symptoms of depression: unmet needs. J Clin Psychiatry. 2003;64 Suppl 7:5-11.

5. Husain MM, Rush AJ, Trivedi MH, et al. Pain in depression: STAR*D study findings. $J$ Psychosom Res. 2007;63(2):113-122.

6. Reed C, Monz BU, Perahia DG, et al. Quality of life outcomes among patients with depression after 6 months of starting treatment: results from FINDER. $J$ Affect Disord. 2009;113(3):296-302.

7. Ghaemi SN. Why antidepressants are not antidepressants: STEP-BD, STAR*D, and the return of neurotic depression. Bipolar Disord. 2008; 10(8):957-968.

8. Angst J, Adolfsson R, Benazzi F, et al. The HCL-32: towards a selfassessment tool for hypomanic symptoms in outpatients. JAffect Disord. 2005;88(2):217-233.

9. Perugi G, Canonico PL, Carbonato P, et al. Unexplained somatic symptoms during major depression: prevalence and clinical impact in a national sample of Italian psychiatric outpatients. Psychopathology. 2011;44(2):116-124.

10. Zung WW. A self-rating depression scale. Arch Gen Psychiatry. 1965; 12:63-70.

11. Zung WW. A rating instrument for anxiety disorders. Psychosomatics. 1971;12(6):371-379.
Neuropsychiatric Disease and Treatment

\section{Publish your work in this journal}

Neuropsychiatric Disease and Treatment is an international, peerreviewed journal of clinical therapeutics and pharmacology focusing on concise rapid reporting of clinical or pre-clinical studies on a range of neuropsychiatric and neurological disorders. This journal is indexed on PubMed Central, the 'PsycINFO' database and CAS, and is the official

\section{Dovepress}

journal of The International Neuropsychiatric Association (INA). The manuscript management system is completely online and includes a very quick and fair peer-review system, which is all easy to use. Visit http://www.dovepress.com/testimonials.php to read real quotes from published authors. 